\title{
Preliminary Investigations on Distinct Proteomics Profiles for Different Subtypes of Non-Syndromic Cleft Palate Based on SELDI-TOF-MS Hong He ${ }^{1,2 \#}$, Xueting Wang ${ }^{1}$, Xinyue Zhang1, Ying Wang ${ }^{1}$, Guoyun Wang ${ }^{3 * *}$, Feiyun Ping ${ }^{3 *}$ \\ ${ }^{1}$ The Affiliated Stomatology Hospital, Zhejiang University School of Medicine, Hangzhou, China. \\ ${ }^{2}$ Key Laboratory of Oral Biomedical Research of Zhejiang Province, Hangzhou China. \\ ${ }^{3}$ Affiliated Second Hospital, School of Medicine, Zhejiang University, China. \\ *Co-Corresponding Authors: Guoyun Wang, Feiyun Ping. \\ ${ }^{\#}$ Co-first Authors: Hong He, Guoyun Wang.
}

\section{Abstract}

Cleft lip and palate usually affect patients in speech, hearing, appearance and psychology, and they need multidisciplinary care from birth to adulthood. However, it is still difficult to make a clear and early diagnosis of cleft palate during pregnancy. The study of differential proteomics will increase our understanding for the pathogenesis and manifestations of cleft palate, and help find methods to assist in early screening, prevent or even in treating cleft palate. Thirty clinical patients with cleft lip and palate and thirty normal controls were collected, the protein expression between different groups was detected by SELDI-TOF-MS technology, and the differentially expressed proteins were analyzed. In between the cleft palate only (CPO) group and the normal control group, the differently expressed protein peaks observed were 4180.3542 and 5639.2742. In between the CPO group and the complete cleft palate and lip group, the peaks were 3291.2347, 4812.2454, 4969.911 and 7791.0462. And in between the unilateral cleft palate and lip group and the bilateral cleft palate and lip group, the peaks were 2529.6336 and 5069.8178. These results may benefit the research on the etiology and early detection of non-syndromic cleft palate.

Keywords: Non-syndromic cleft palate; SELDI-TOF-MS; Proteomics; Etiology

\section{INTRODUCTION}

Cleft palate and lip are the most common congenital deformities of the oral and maxillofacial region, arising in approximately 1.7 per 1000 live births worldwide with ethnic and geographic variation [1]. Cleft palate can occur alone or concurrently with cleft lip and can be classified into cleft palate only (CPO), unilateral cleft palate and lip, and bilateral cleft palate and lip [2]. Children suffering these disorders usually have problems with speaking, feeding, hearing, appearance and psychology, and need multidisciplinary care from birth to adulthood [1,3]. While some cleft palate is part of genetic syndrome with others systemic diseases or malformations such as congenital heart defects (31.1\%), deformations (22.4\%), hydrocephaly $(11.2 \%)[4]$, the $50-70 \%$ of orofacial clefts are nonsyndromic clefts, which are caused by multiple factors including genetic factors with highly genetically heterogeneous and environmental risk factors [5].

In recent years, with the popularization of routine examinations during pregnancy and the improvement of the performance of ultrasound imaging technology, fetuses with cleft lip can be detected through early pregnancy tests, and the pregnancy can be terminated in time. However, it's still a challenge to make a clear diagnosis of cleft palate during pregnancy with reporting errors range from $10-60 \%$, because palate is an internal structure which can be easily shadowed by the surrounding bones and it's largely associated with characterization of the secondary palate $[5,6]$. Nevertheless, the detecting accuracy is improving with newer technologies, such as three-dimensional ultrasound using modern information technology [7]. Moreover, the recurrence of cleft is quite common and unpredictable. 
Preliminary Investigations on Distinct Proteomics Profiles for Different Subtypes of Non-Syndromic Cleft Palate Based on SELDI-TOF-MS

Palatal development is a complex process involving different gene expression, a network of signaling pathways, transcription factors and cell-surface receptors, which are manifested via proteins. Lulin Huang et al. [8] identified several genes/loci associated to non-syndromic orofacial cleft (NSOFC) by mateanalysis, including IRF6, PAX9, DLK1, POMGNT2, WHSC1, DOCK9, FOXC2-FOXL1, MAU2 for CPO, IRF6, $M Y C N, V A X 1$ and $M A F B$ for cleft palate with cleft lip and revealed that developmental transcription factors (TFs) played important roles in the regulation of orofacial cleft. Several animal model studies have indicated some molecular pathways relevant to proper closure of the palate, such as BMP, TGF- $\beta$, SHH, FGF signaling pathways [2].

With the development and application of proteomics technology, it's possible to analyze the proteins expressed differentially by large-scale high-throughput screening, and discover the relevant characteristic proteins in the process of cleft palate, which can increase our understanding about the etiology of non-syndromic cleft palate and improve prevention, diagnosis, treatment, and prognosis for patients with orofacial cleft. The proteomics technology, surface enhanced laser desorption/ionization time-of-flight mass spectrometry (SELDI-TOF-MS) [9,10], can detect the molecular weight of ionized protein and draw a mass spectrum with high sensitivity. Comparing the protein profiles of different groups, we may find specific proteins related to the disease.

Therefore, in our research, we used SELDI-TOF-MS to screen the differential serum protein between cleft patients and normal human controls, which may benefit the research on the etiology of non-syndromic cleft palate.

\section{METHODS}

\section{Clinical Date}

Thirty cleft pediatric patients (18 males, 12 females) who were presented and treated at Department of Oral and Maxillofacial Surgery, the Second Affiliated Hospital of Zhejiang University School of Medicine from March 2005 to January 2006, were recruited, excluding patients with syndromes as we know, such as Van der Woude syndrome, Robin's syndrome, Meckel syndrome, velo-cardiofacial syndrome (VCFs). The study group was divided into 4 subtypes, including cleft lip only, cleft palate only, unilateral cleft palate and lip, and bilateral cleft palate and lip. Meanwhile, 30 children (18 males, 12 females), from healthy children outpatients for regular oral examination were recruited and set as healthy control group. All the subjects aged between 0.3-6 years, and there is no biological kinship among them. All patients and healthy controls participating in the experiment have understood the relevant content and obtained a signed, written, informed consent.

\section{Collection and Disposition of Serum Samples}

We drew $4 \mathrm{~mL}$ fasting peripheral blood of all the subjects in the early morning, and the blood specimens were put at room temperature for $0.5-1 \mathrm{~h}$. Then the sera was separated by $20-30 \mathrm{~min}$ centrifugation (3000 $\mathrm{r} / \mathrm{min}$ ), then drawn into an EP tube with label and placed in $-20^{\circ} \mathrm{C}$ refrigerator.

\section{Laboratory Procedures of Serum Samples}

After thawing the serum samples at room temperature and $5 \mathrm{~min}$ centrifugation $\left(3000 \mathrm{r} / \mathrm{min}, 4^{\circ} \mathrm{C}\right), 10 \mu \mathrm{L}$ sample was diluted with $90 \mu \mathrm{L} 0.5 \%$ U9 buffer (9 mol/L urea, 0.15\% CHAPS (3-[(3-cholamidopropyl) dimethylammonio]-1-propanesulfonate), pH7.14), then added to albumin affinity resin (CibacronBlue 3GA, Sigma), shaking at $600 \mathrm{r} / \mathrm{min}$ and reacting for $60 \mathrm{~min}$ at $4^{\circ} \mathrm{C}$. After $5 \mathrm{~min}$ centrifugation $(2000 \mathrm{r} / \mathrm{min}$, $4^{\circ} \mathrm{C}$ ), $50 \mu \mathrm{L}$ supernatant was diluted to $200 \mu \mathrm{L}$ with HEPES (20mmol/L, pH7.14), and added to a 96-well Bioprocessor with $\mathrm{H} 4$ chip installed (Ciphergen) for $60 \mathrm{~min}$ oscillatory reaction $\left(600 \mathrm{r} / \mathrm{min}, 4^{\circ} \mathrm{C}\right)$, then discarded the supernatant. Wash the chip with $200 \mu \mathrm{L}$ HEPES (20mmol/L, pH7.14) 3 times and 5min each time. Wash the chip with deionized water 2 times and 1 min each time. Add $0.15 \mu$ L energy absorbing molecule CHCA (Ciphergen) to the chip after drying and repeat. Finally dry the chip ready for later experiment.

\section{SELDI-TOF-MS Biotechnology}

Test the equipment operation and detector sensitivity. Correct the accuracy and make the error rate less than $0.1 \%$ using the peptide molecular mass standard (Ciphergen Biosystems). Set parameters as follows: the maximum protein molecular weight was 30000 , the range of peaks detected automatically was $2000 \sim 30000 \mathrm{~m} / \mathrm{z}$, the laser intensity was 135 and 65 laser shots for per sample.

The protein chip plate containing the detection samples was put into the mass spectrometer, and 
Preliminary Investigations on Distinct Proteomics Profiles for Different Subtypes of Non-Syndromic Cleft Palate Based on SELDI-TOF-MS

SELDI Protein Biology System II plus (PBS II plus) was used to collect the data. ProteinChip Software (Version 3.0, Ciphergen Biosystems) was used to correct the original data to make the total ion intensity and molecular weight of the data uniform. Then filter noise by setting the initial noise filter value to 2.2 and the secondary to 1.0 Biomarker Wizard (Ciphergen, USA) software was used for preliminary screening of differential proteins, which $\mathrm{m} / \mathrm{z}$ peaks existed in more than $10 \%$ of samples with the deviation of the same protein $\mathrm{m} / \mathrm{z}$ peak in different samples less than $0.3 \%$.

\section{Statistical Analysis}

Kruskal-Wallis multivariate analysis of variance used to analyze the data of different groups to find protein

Table1. Differential protein peak values peaks with differential expression between groups.

\section{RESULTS}

The statistically differential protein peak values of mass to charge ratio $(\mathrm{m} / \mathrm{z}$ ) were observed (table 1$)$. In between the cleft palate only group and the normal control group, the differently expressed protein peaks observed were 4180.3542 and 5639.2742 . In between the cleft palate only group and the cleft palate and lip group, the peaks were 3291.2347, 4812.2454, 4969.911 and 7791.0462. In between the unilateral cleft palate and lip group and the bilateral cleft palate and lip group, the peaks were 2529.6336 and 5069.8178.

\begin{tabular}{|l|l|}
\hline \multicolumn{1}{|c|}{ Groups } & \multicolumn{1}{|c|}{ Differential protein peak values (p<0.01) } \\
\hline Cleft palate only vs Normal controls & $4180.3542,5639.2742$ \\
\hline Cleft palate only vs Cleft palate and lip & $3291.2347,4812.2454,4969.911,7791.0462$ \\
\hline Unilateral cleft palate and lip vs Bilateral cleft palate and lip & $2529.6336,5069.8178$ \\
\hline
\end{tabular}

\section{Discussion}

In our study, the peak intensity reflects the content difference of protein with the same PSR between two or more groups, and $\mathrm{P}$ value indicates the degree of difference. The differential protein peaks suggest the existence of differential functional proteins or peptides between different groups, and that the subtypes of non-syndromic cleft palate are affected by various genetic genes. Lulin Huang et al. [8] have identified 18 genes/loci responsible for NSOFC, nine genes/loci (IRF6, PAX9, DLK1, POMGNT2, WHSC1, DOCK9, FOXC2-FOXL1, MAU2) were associated with CPO, seven genes/loci (IRF6, DLK1, MYCN, VAX1, MAFB, GRM5, ALX1) were associated with cleft lip only (CLO), and four genes/loci (IRF6, MYCN, VAX1, MAFB) were associated with cleft lip and palate, from which we can find IRF6 and DLK1 were related to both CPO and CLO. So, our results can be the supporting evidence for the different mechanisms of cleft palate with cleft lip and $\mathrm{CPO}$ on the protein pathway and different structural proteins.

The down-regulated expression dosage of IRF6 is a risk factor for $\mathrm{CPO}$ but is protective for CLO oppositely $[11,12]$. The developmental TFs play critical roles in the pathogenesis of CLO/CPO. And it's necessary to do further identification of the protein and confirm the role of proteins on the occurrence and development of cleft palate. After the emergence of proteomics technology, differential protein screening proteomics, such as SELID-TOF or combined with LCMS, have provided new technical support for the functional study of disease genes, proteins, cell functions, clinical manifestations.

The principle of SELDI technology is based on the surface-enhanced adsorption of special chips. The chip firstly undergoes special treatment to directly drop the sample to be tested on the chip surface (the sample to be tested can be derived from serum, urine, secretion, cell lysate, tissue, etc.), then specific proteins in the sample are adsorbed to the chip by affinity. On the surface of the solid phase matrix, the impurity protein and other contaminants on the chip are washed away with a buffer solution, and then an energy absorbing molecular (EAM) is added to the chip. After the chip is dried, it is bombarded with a laser in a vacuum tube. After absorbing energy, the protein is resolved and ionized. It is detached from the surface of the chip under the force of an electric field and flew toward the cathode, where it is detected by an ion detector. Due to the different mass-to-charge ratios $(\mathrm{m} / \mathrm{z})$ of these ions, the flight time in the vacuum tube is also different. Among them, the small flight time is short, the fastest flight and the first to reach the ion detector is detected. 
Preliminary Investigations on Distinct Proteomics Profiles for Different Subtypes of Non-Syndromic Cleft Palate Based on SELDI-TOF-MS

SELDI software can process and analyze mass spectrum information quickly. By comparing the maps of normal people with those of patients with a certain disease, we can find and capture the disease-specific proteins. In the past, chromatography, two-dimensional electrophoresis, spectroscopy, mass spectrometry and other techniques were used in protein research. These technologies are notsuitable for large-scale population screening and clinical examination because of their high requirements, expensive instruments, tedious steps and long time-consuming. SELDI-TOF-MS technology combines the specific protein chip system and mass spectrometer. The sample does not need to be purified in advance. It can directly add the samples of serum, urine, tissue and cell lysate to the chip for detection. The obtained map is simple and repeatable. It can detect the protein with molecular weight of 200 $500 \mathrm{kd}$, especially for the detection of low abundance protein or polypeptide. The analysis of a sample can be completed in tens of minutes, and the amount of information processed is far greater than that of twodimensional electrophoresis. This technology can be used for protein quantitative analysis which can not be carried out by MALDI [3]. It can analyze a small number of samples without pretreatment with high throughput, high efficiency and high sensitivity, and is suitable for basic medical research, clinical diagnosis and large-scale population screening [4].

Children aged 0.3-6 years were selected, avoiding significant changes in the hormone and body protein content after puberty [13]. Meanwhile familial cleft lip and palate patients were excluded, reducing genetic interference and improving the reliability of differential protein research. Provided that the relevant characteristic proteins can be discovered in the process of cleft lip and palate, the treatment and interference of cleft lip and palate can be guided on the protein pathway. Through the reconstruction of protein function, the clinical performance of the cleft lip and palate can stay or turn to the normal sequence.

However, due to the limited sample size, there's a lack of statistical significance for the comparison of protein spectrum differences. More samples and further intensive studies are needed.

\section{CONCLUSION}

This paper reports the differential serum proteome between cleft palate and normal, cleft palate only and with cleft lip, unilateral and bilateral cleft palate and lip, hopefully providing some data for the study on pathogenesis of cleft.

\section{ACKNOWLEDGEMENT}

The work was supported by the Teaching Reform Project of Zhejiang University School of Medicine yxyb20172030. National and provincial Health Commission Co-construction Fund WKJ-ZJ-1623. National Health Commission Public Welfare Fund (201502018). National Key R\&D Program of China 2016YFC0902702. 2016 Fund for 151 talents second level Zhejiang Province 2014-2017. Baoshi Oversea Scholarship zups1507 2013-2015. China Scholarship Council [2009]3004. Scientific Research Foundation for the Returned Oversea Chinese Scholar J20120036 from HR Office of Zhejiang Province.

\section{Consent and EThical Approval}

According to international standard, patients' written consent and ethical approval have been collected and preserved by the authors.

\section{REFERENCES}

[1] Mossey PA, Little J, Munger RG, et al. Cleft lip and palate. Lancet. 2009 Nov 21; 374 (9703): 1773-85.

[2] Burg ML, Chai Y, Yao CA, et al. Epidemiology, Etiology, and Treatment of Isolated Cleft Palate. Front Physiol. 2016; 7: 67.

[3] Leslie EJ, Marazita ML. Genetics of cleft lip and cleft palate. Am J Med Genet C Semin Med Genet. 2013 Nov;163C(4):246-258.

[4] P. Mossey, E. Catilla. Global Registry and Database on Craniofacial Anomalies: Report of a WHO Registry Meeting on Craniofacial Anomalies. Geneva:WorldHealth Organization.2003.https:// apps.who.int/iris/handle/10665/42840

[5] Tutschek B, Blaas HK. 3D ultrasound and the fetal palate. Re: Qualitative evaluation of Crystal Vue rendering technology in assessment of fetal lip and palate. Ultrasound Obstet Gynecol 2017; 50: $274-277$

[6] Steinberg JP, Gosain AK. Thirty Years of Prenatal Cleft Diagnosis: What Have We Learned? Plast Reconstr Surg. 2015 Sep;136(3):550-7. 
Preliminary Investigations on Distinct Proteomics Profiles for Different Subtypes of Non-Syndromic Cleft Palate Based on SELDI-TOF-MS

[7] Wójtowicz A, Wójtowicz W, Jurek J, et al. Evaluation of the fetal palate at 11 to $13(+6)$ weeks of gestation based on an analysis of static ultrasound images using modern IT techniques. Prenat Diagn. 2018 May;38(6):414-421.

[8] Huang L, Jia Z, Shi Y, et al. Genetic factors define CPO and CLO subtypes of nonsyndro micorofacial cleft. PLoS Genet. 2019 Oct 14; 15(10):e1008357.

[9] Seibert V, Wiesner A, Buschmann T, et al. Surfaceenhanced laser desorption ionization time-offlight mass spectrometry (SELDI TOF-MS) and ProteinChip technology in proteomics research. Pathol Res Pract. 2004;200(2):83-94.

[10] Gemoll T, Roblick UJ, Auer G, et al. SELDI-TOF serum proteomics and colorectal cancer: a current overview. Arch Physiol Biochem. 2010 Oct-Dec;116(4-5):188-96.

[11] Zucchero TM, Cooper ME, Maher BS, et al. Interferon Regulatory Factor 6 (IRF6) Gene Variants and the Risk of Isolated Cleft Lip or Palate. N Engl J Med 2004; 351:769-780

[12] Mijiti A, Ling W, Guli, et al. Association of singlenucleotide polymorphisms in the IRF6 gene with non-syndromic cleft lip with or without cleft palate in the Xinjiang Uyghur population. British Journal of Oral and Maxillofacial Surgery, 2015, 53(3):268-274.

[13] He H, Zhang MY, Wang Y, et al. Differencial Serum Proteomes between Subphenotypes of Nonsyndromic Cleft Lip with or without Cleft Palate. Archives of Dentistry and Oral Health V2. I2. 2019, 2(2):24-28

Citation: Hong He", Xueting Wang, Xinyue Zhang, Ying Wang, Guoyun Wang ${ }^{* *}$, Feiyun Ping*. Preliminary Investigations on Distinct Proteomics Profiles for Different Subtypes of Non-Syndromic Cleft Palate Based on SELDI-TOF-MS. Archives of Dentistry and Oral Health. 2020; 3(1): 01-05.

Copyright: (C) 2020 Hong He, Xueting Wang, Xinyue Zhang, Ying Wang, Guoyun Wang, Feiyun Ping. This is an open access article distributed under the Creative Commons Attribution License, which permits unrestricted use, distribution, and reproduction in any medium, provided the original work is properly cited. 